\title{
Non-perturbative Heavy Quark Effective Theory: a test and its matching to QCD ${ }^{1}$
}

\author{
Rainer Sommer \\ DESY, Platanenallee 6, 15738 Zeuthen, Germany
}

\begin{abstract}
We give an introduction to the special problems encountered in a treatment of HQET beyond perturbation theory in the gauge coupling constant. In particular, we report on a recent test of HQET as an effective theory for QCD and discuss how HQET can be implemented on the lattice including the non-perturbative matching of the effective theory to QCD.
\end{abstract}

Keywords: Heavy quark effective theory; Non-perturbative renormalization; Matching; Lattice QCD; Static approximation; Mass of the b-quark

PACS: 11.10.Gh; 11.15.Ha; 12.15.Hh; 12.38.Gc; 12.39.Hg; 14.65.Fy

\section{INTRODUCTION}

Heavy quark effective theory is routinely used in phenomenology. In these applications, the matching to QCD is achieved perturbatively and matrix elements of the operators in the effective theory are determined from experiment and models. However, HQET took its origin as an effective theory in the lattice regularization, where it was designed as a solution to the problem of treating quarks which are heavy compared to the inverse lattice spacing and thus do not propagate properly in the standard relativistic framework [1].

Unfortunately, after considerable initial activity (see e.g. [2, 3, 4] and references therein) the non-perturbative treatment of the effective theory on the lattice had been somewhat dormant for a while. The reason is that it was realized [5] that a nonperturbative matching to QCD is needed; otherwise the continuum limit does not exist. A practicable solution of this problem was only found recently [6, 7, 8].

Here we point out that a non-perturbative matching is necessary on and off the lattice, the problem being most severe on the lattice and we review a non-perturbative test of HQET. We then explain a recent strategy to perform fully non-perturbative computations in HQET and discuss the status and perspectives of this approach.

${ }^{1}$ Invited talk at "6th Conference on Quark Confinement and the Hadron Spectrum”, Sept. 21-25 2004, Villasimius, Italy 


\section{HQET AS AN ASYMPTOTIC EXPANSION OF QCD}

Consider QCD at energies low enough such that the top-quark may be neglected altogether. In the QCD Lagrangian

$$
\mathscr{L}_{\mathrm{QCD}}=-\frac{1}{2 g_{0}^{2}} \operatorname{tr}\left\{F_{\mu v} F_{\mu v}\right\}+\sum_{f} \bar{\psi}_{f}\left[D_{\mu} \gamma_{\mu}+m_{f}\right] \psi_{f}
$$

the sum over flavors then extends over $f=\mathrm{u}, \mathrm{d}, \mathrm{s}, \mathrm{c}, \mathrm{b}$. An effective theory, HQET is expected to provide the asymptotic expansion of a certain (large) set of energies (e.g. mass splittings) and matrix elements of QCD in terms of the inverse of the mass of the b-quark. ${ }^{2}$ We restrict our discussion to the energies and matrix elements of states which contain a single b-quark at rest and refer to reviews such as [9, 10] for the general case of finite velocity. The Lagrangian of the theory, which may be obtained by a formal $1 / m_{\mathrm{b}}$ expansion (see e.g. [11]), is then given by the replacement

$$
\bar{\psi}_{\mathrm{b}}\left[D_{\mu} \gamma_{\mu}+m_{\mathrm{b}}\right] \psi_{\mathrm{b}} \rightarrow \mathscr{L}_{\text {stat }}+\mathscr{L}^{(1)}+\ldots, \quad \mathscr{L}_{\text {stat }}=\bar{\psi}_{\mathrm{h}}\left[D_{0}+\delta m\right] \psi_{\mathrm{h}}
$$

Here $\mathscr{L}^{(1)}$ is of order $1 / m_{\mathrm{b}}$ and the mass term of the b-quark has been removed from the Lagrangian such that observable quantities in the b-sector have a finite limit as $m_{\mathrm{b}} \rightarrow \infty$ (with a suitable counter term $\delta m$ ). The effective heavy quark field $\psi_{\mathrm{h}}$ has only two degrees of freedom as appropriate for a non-relativistic spin 1/2 particle. Still it is notationally convenient to keep $\psi_{\mathrm{h}}$ as a 4-component spinor but impose the constraint

$$
P_{+} \psi_{\mathrm{h}}=\psi_{\mathrm{h}}, P_{+}=\left(1+\gamma_{0}\right) / 2 ;
$$

i.e. the lower components vanish in the Dirac representation. In order to discuss matrix elements, such as the B-meson decay constant, also the composite fields involving bquarks are translated to the effective theory, for example:

$$
A_{0}=Z_{\mathrm{A}} \bar{\psi}_{1} \gamma_{0} \gamma_{5} \psi_{\mathrm{b}} \quad \rightarrow \quad A_{0}^{\text {stat }}=Z_{\mathrm{A}}^{\mathrm{stat}} \bar{\psi}_{1} \gamma_{0} \gamma_{5} \psi_{\mathrm{h}}
$$

Here $Z_{\mathrm{A}}, Z_{\mathrm{A}}^{\text {stat }}$ are the renormalization constants of the composite fields.

The effective theory is valid for the low-lying energy levels as well as their matrix elements, the simplest one being

$$
\Phi^{\mathrm{QCD}} \equiv F_{\mathrm{B}} \sqrt{m_{\mathrm{B}}}=Z_{\mathrm{A}}\left\langle 0\left|A_{0}\right| B\right\rangle .
$$

It is scale independent, due to the chiral symmetry of QCD in the massless limit (including $m_{\mathrm{b}}=0$ ). In the effective theory this symmetry is absent and $Z_{\mathrm{A}}^{\text {stat }}$ depends on the energy scale, $\mu$, used in the renormalization condition which defines the finite current. Instead of $\Phi^{\text {stat }}(\mu) \equiv Z_{\mathrm{A}}^{\text {stat }}(\mu)\left\langle 0\left|A_{0}\right| B\right\rangle_{\text {stat }}$ it is therefore better to consider the renormalization group invariant matrix element

$$
\Phi_{\mathrm{RGI}}=\lim _{\mu \rightarrow \infty}\left[2 b_{0} \bar{g}^{2}(\mu)\right]^{-\gamma_{0} / 2 b_{0}} \Phi^{\mathrm{stat}}(\mu) .
$$

\footnotetext{
${ }^{2}$ Powers of $1 / m_{\mathrm{b}}$ are understood to be accompanied by slowly (logarithmically) varying functions of $m_{\mathrm{b}}$.
} 
It is both $\mu$ and renormalization scheme independent, as is easily seen using $\Phi_{\text {scheme }}^{\text {stat }}(\mu)=\Phi_{\text {scheme }}^{\text {stat }}(\mu)\left(1+\mathrm{O}\left(\bar{g}^{2}(\mu)\right)\right.$. In eq. (6), the coefficients $b_{0}, \gamma_{0}$ defined by

$$
\beta(\bar{g}) \equiv \mu \frac{\mathrm{d}}{\mathrm{d} \mu} \bar{g}=-b_{0} \bar{g}^{3}+\mathrm{O}\left(\bar{g}^{5}\right), \quad \gamma(\bar{g}) \equiv \frac{\mu}{Z_{\mathrm{A}}^{\text {stat }}} \frac{\mathrm{d}}{\mathrm{d} \mu} Z_{\mathrm{A}}^{\text {stat }}=-\gamma_{0} \bar{g}^{2}+\mathrm{O}\left(\bar{g}^{4}\right)
$$

enter. We can now write down the HQET-expansion of the QCD matrix element

$$
\begin{aligned}
\Phi^{\mathrm{QCD}} & =C_{\mathrm{PS}}\left(M_{\mathrm{b}} / \Lambda_{\overline{\mathrm{MS}}}\right) \times \Phi_{\mathrm{RGI}}+\mathrm{O}\left(1 / M_{\mathrm{b}}\right), \\
M_{\mathrm{b}} & =\lim _{\mu \rightarrow \infty}\left[2 b_{0} \bar{g}(\mu)\right]^{-d_{0} / 2 b_{0}} \bar{m}(\mu), \quad \tau(\bar{g}) \equiv \frac{\mu}{\bar{m}} \frac{\mathrm{d}}{\mathrm{d} \mu} \bar{m}=-d_{0} \bar{g}^{2}+\ldots \\
\Lambda_{\overline{\mathrm{MS}}} & =\lim _{\mu \rightarrow \infty} \mu\left(b_{0} \bar{g}_{\overline{\mathrm{MS}}}^{2}(\mu)\right)^{-b_{1} /\left(2 b_{0}^{2}\right)} \mathrm{e}^{-1 /\left(2 b_{0} \bar{g}_{\overline{\mathrm{MS}}}^{2}(\mu)\right)} .
\end{aligned}
$$

Let us dicuss the somewhat unfamiliar form of eq. (8) and the conversion function $C_{\mathrm{PS}}\left(M_{\mathrm{b}} / \Lambda_{\overline{\mathrm{MS}}}\right)$. In a more conventional form we have

$$
\Phi^{\mathrm{QCD}}=C_{\text {match }}\left(m_{\mathrm{b}} / \mu\right) \times \Phi_{\overline{\mathrm{MS}}}(\mu)+\mathrm{O}\left(1 / m_{\mathrm{b}}\right)
$$

with a matrix element $\Phi_{\overline{\mathrm{MS}}}(\mu)$ renormalized in the effective theory in the $\overline{\mathrm{MS}}$ scheme and the matching coefficient $C_{\mathrm{match}}\left(m_{\mathrm{b}} / \mu\right)$ depending on the b-quark mass $m_{\mathrm{b}}$ in the $\overline{\mathrm{MS}}$ scheme at scale $m_{\mathrm{b}}$, i.e. $\bar{m} \overline{\mathrm{MS}}\left(m_{\mathrm{b}}\right)=m_{\mathrm{b}}$. The factor $C_{\text {match }}$ is determined (usually in perturbation theory) such that eq. (11) holds for some particular matrix element of the current and will then be valid for all matrix elements. Contact to eq. (8) is easily made by using

$$
\frac{\Phi_{\mathrm{RGI}}}{\Phi_{\overline{\mathrm{MS}}}(\mu)}=\left[2 b_{0} \bar{g}^{2}(\mu)\right]^{-\gamma_{0} / 2 b_{0}} \exp \left\{-\int_{0}^{\bar{g}(\mu)} \mathrm{d} g\left[\frac{\gamma_{\overline{\mathrm{MS}}}(g)}{\beta_{\overline{\mathrm{MS}}}(g)}-\frac{\gamma_{0}}{b_{0} g}\right]\right\},
$$

setting the arbitrary renormalization point $\mu$ to $m_{\mathrm{b}}$ and identifying

$$
\begin{aligned}
C_{\mathrm{PS}}\left(\frac{M_{\mathrm{b}}}{\Lambda_{\overline{\mathrm{MS}}}}\right) & =C_{\text {match }}(1) \frac{\Phi_{\overline{\mathrm{MS}}}\left(m_{\mathrm{b}}\right)}{\Phi_{\mathrm{RGI}}} \\
& =\left[2 b_{0} \bar{g}^{2}\left(m_{\mathrm{b}}\right)\right]^{\gamma_{0} / 2 b_{0}} \exp \left\{\int_{0}^{\bar{g}\left(m_{\mathrm{b}}\right)} \mathrm{d} g\left[\frac{\gamma_{\text {match }}(g)}{\beta_{\overline{\mathrm{MS}}}(g)}-\frac{\gamma_{0}}{b_{0} g}\right]\right\},
\end{aligned}
$$

where $\bar{g}$ is taken in the $\overline{\mathrm{MS}}$ scheme. The last equation may be taken as a definition of the anomalous dimension $\gamma_{\text {match }}$ in the "matching scheme". It has contributions from $\gamma_{\overline{\mathrm{MS}}}$ as well as from $C_{\text {match }}=1+c_{1} \bar{g}^{2}+\ldots$, namely

$$
\gamma_{\text {match }}(\bar{g})=-\gamma_{0} \bar{g}^{2}-\left[\gamma_{1}^{\overline{\mathrm{MS}}}+2 b_{0} c_{1}\right] \bar{g}^{4}+\ldots
$$

Note that replacing the $\overline{\mathrm{MS}}$ coupling by a non-perturbative one, $\gamma_{\text {match }}$ may also be defined beyond perturbation theory through eqs. (13)8). ${ }^{3}$ Another advantage of eq. (13)

${ }^{3}$ Clearly the r.h.s. of eq. (13) is a function of $\bar{g}^{2}\left(m_{\mathrm{b}}\right)$, i.e. a function of $m_{\mathrm{b}} / \Lambda_{\overline{\mathrm{MS}}}$. We prefer to write it as a function of the ratio of renormalization group invariants, $M_{\mathrm{b}} / \Lambda_{\overline{\mathrm{MS}}}$. 
is that $C_{\mathrm{PS}}$ is independent of the arbitrary choice of renormalization scheme for the effective operators in the effective theory. Apart from the choice of the QCD coupling, the "convergence" of the series eq. (14) is dictated by the physics, nothing else. Note further that (at leading order in $1 / M$ ) the conversion function $C_{\mathrm{PS}}$ contains the full (logarithmic) mass-dependence. The non-perturbative effective theory matrix elements are mass independent numbers. Conversion functions such as $C_{\mathrm{PS}}$ are universal for all (low energy) matrix elements of their associated operator. Thus

$$
Z_{\mathrm{A}}^{2}\left\langle A_{0}^{\dagger}(x) A_{0}(0)\right\rangle_{\mathrm{QCD}} \stackrel{x^{2} \gg 1 / M_{\mathrm{b}}^{2}}{\sim}\left[C_{\mathrm{PS}}\left(\frac{M_{\mathrm{b}}}{\Lambda_{\overline{\mathrm{MS}}}}\right)\right]^{2}\left\langle A_{0}^{\text {stat }}(x)^{\dagger} A_{0}^{\mathrm{stat}}(0)\right\rangle_{\mathrm{RGI}}+\mathrm{O}\left(\frac{1}{M_{\mathrm{b}}}\right)
$$

is a straight forward generalization of eq. (8).

Analogous expressions for the conversion functions are valid for the time component of the axial current replaced by other composite fields, for example the space components of the vector current. Based on the work of [12, 13, 14] and recent efforts their perturbative expansion is known including the 3-loop anomalous dimension $\gamma_{\text {match }}$ obtained from the 3-loop anomalous dimension $\gamma_{\overline{\mathrm{MS}}}$ [15] and the 2-loop matching function

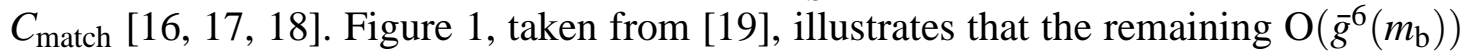
errors in $C_{\mathrm{PS}}$ seem to be relatively small.

Although it is generally accepted that HQET is an effective theory of QCD in the sense that was just described, tests of this equivalence are rare and mostly based on phenomenological analysis of experimental results. A pure theory test can be performed if QCD including a heavy enough quark can be simulated on the lattice at lattice spacings which are small enough to be able to take the continuum limit. This has recently been achieved [19] and will be summarized below.

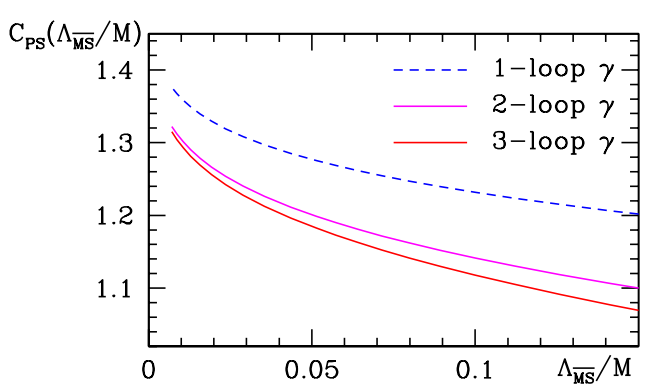

1: $C_{\mathrm{PS}}$ estimated in perturbation theory.

\subsection{Tests of HQET in a finite volume}

We start with the QCD side of such a test. Lattice spacings such that $a m_{\mathrm{b}} \ll 1$ can be reached if one puts the theory in a finite volume, $L^{3} \times T$ with $L, T$ not too large. We shall use $T=L$. For various practical reasons, so-called Schrödinger functional boundary conditions are chosen, i.e. Dirichlet in time (at $\left.x_{0}=0, T\right)$ and periodic in space [20,21]. Equivalent boundary conditions are easily imposed in the effec-

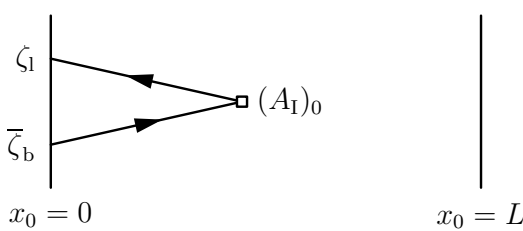

2: The correlation function $f_{\mathrm{A}}$. tive theory [22]. We then form correlation functions of boundary quark fields $\zeta$ (located at $\left.x_{0}=0\right)$ and composite fields such as the time component of the axial current in the 

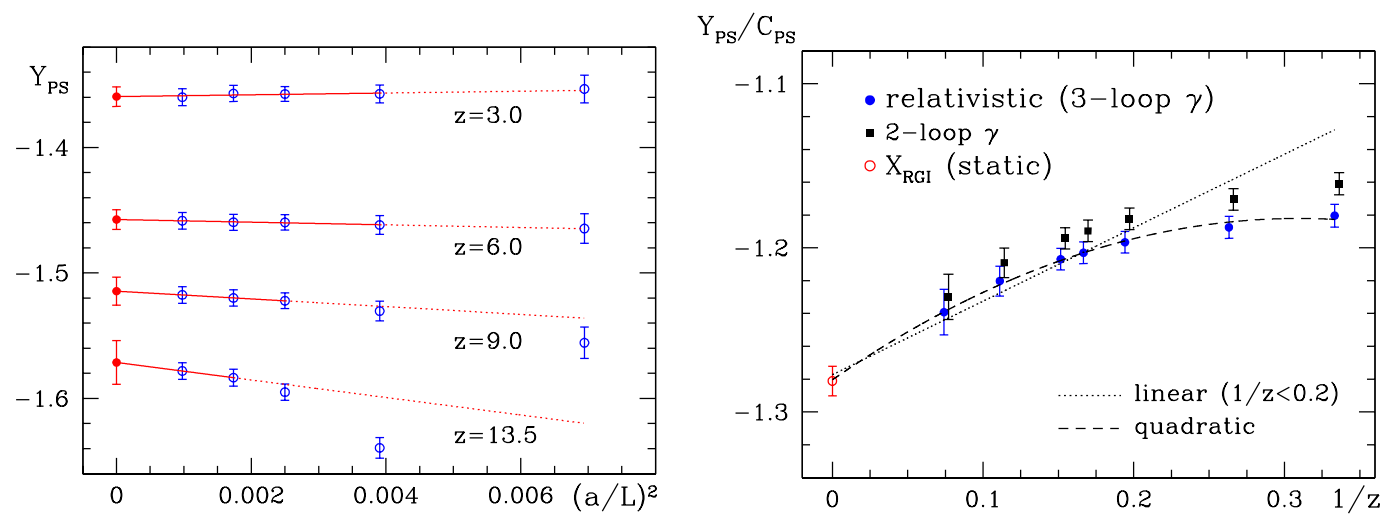

FIGURE 3. Testing eq. (19) through numerical simulations in the quenched approximation and for $L \approx 0.2 \mathrm{fm}[19]$. The physical mass of the b-quark corresponds to $z \approx 5$.

bulk $\left(0<x_{0}<T\right)$, as illustrated in Fig. 2 and given for example by

$$
f_{\mathrm{A}}\left(x_{0}\right)=-\frac{a^{6}}{2} \sum_{\mathbf{y}, \mathbf{z}}\left\langle\left(A_{\mathrm{I}}\right)_{0}(x) \bar{\zeta}_{\mathrm{b}}(\mathbf{y}) \gamma_{5} \zeta_{\mathrm{l}}(\mathbf{z})\right\rangle .
$$

(The current $A_{\mathrm{I}}$ represents the $\mathrm{O}(a)$-improved version of the axial current for which lattice artifacts linear in the lattice spacing are absent.) Another correlation function, $f_{1}$, describes the propagation of a quark-antiquark pair from the $x_{0}=0$ boundary to the $x_{0}=T$ boundary. For details we refer to [19].

We then take a ratio for which the renormalization factors of the boundary fields cancel,

$$
\begin{aligned}
Y_{\mathrm{PS}}(L, M) \equiv & \left.Z_{\mathrm{A}} \frac{f_{\mathrm{A}}(L / 2)}{\sqrt{f_{1}}}\right|_{T=L}=\frac{\left\langle\Omega(L)\left|A_{0}\right| B(L)\right\rangle}{\||\Omega(L)\rangle\|\||| B(L)\rangle \|}, \\
& |B(L)\rangle=\mathrm{e}^{-L H / 2}\left|\varphi_{\mathrm{B}}(L)\right\rangle,|\Omega(L)\rangle=\mathrm{e}^{-L H / 2}\left|\varphi_{0}(L)\right\rangle .
\end{aligned}
$$

As shown in the above equations, $Y_{\mathrm{PS}}$ can be represented as a matrix element of the axial current between a normalized state $|B(L)\rangle$ with the quantum numbers of a B-meson and $|\Omega(L)\rangle$ which has vacuum quantum numbers. The time evolution $\mathrm{e}^{-L H / 2}$ ensures that both of these states are dominated by energy eigenstates with energies around $2 / L$ and less. In other words, HQET is applicable if $1 / L \ll M$ (and of course $\Lambda \ll M$ ).

One then expects (for fixed $L \Lambda$ )

$$
Y_{\mathrm{PS}}(L, M) / C_{\mathrm{PS}}(M / \Lambda)=X_{\mathrm{RGI}}+\mathrm{O}(1 / z), \quad z=M L,
$$

where the $1 / M$ corrections are written in the dimensionless variable $1 / z$ and $X_{\mathrm{RGI}}$ is defined as $Y_{\text {PS }}$ but at lowest order in the effective theory and renormalized as in eq. (6). Of course such relations are expected after the continuum limit of both sides have been taken separately. For the case of $Y_{\mathrm{PS}}(L, M)$, this is done by the following steps:

- Fix a value $u_{0}$ for the renormalized coupling $\bar{g}^{2}(L)$ (in the Schrödinger functional scheme) at vanishing quark mass. In [19] $u_{0}$ is chosen such that $L \approx 0.2 \mathrm{fm}$. 
- For a given resolution $L / a$, determine the bare coupling from the condition $\bar{g}^{2}(L)=$ $u_{0}$. This can easily be done since the relation between bare and renormalized coupling is known [23].

- Fix the bare quark mass $\widetilde{m}_{\mathrm{q}}$ of the heavy quark such that $L M=z$ using the known renormalization factor $Z_{\mathrm{m}}$ in $M=Z_{\mathrm{m}} \widetilde{m}_{\mathrm{q}}$ [23].

- Evaluate $Y_{\mathrm{PS}}$ and repeat for better resolution $a / L$.

- Extrapolate to the continuum as shown in Fig. 3, left.

As can be seen in the figure, the continuum extrapolation becomes more difficult as the mass of the heavy quark is increased and $\mathrm{O}\left((a M)^{2}\right)$ discretization errors become more and more important. In contrast the continuum extrapolation in the static effective theory (Fig. 4) is much easier (once the renormalization factor relating bare current and RGI current is known [6]). After the continuum limit has been taken, the finite mass QCD observable $Y_{\mathrm{PS}}(L, M)$ turns smoothly into the prediction from the effective theory as illustrated in the r.h.s. figure. Indeed, several such successful tests were performed in [19], one of them free of the perturbative uncertainty in the conversion function (due to reparametrization invariance [24, 25, 26]) and two others with the static $(M \rightarrow \infty)$ limit known from the spin symmetry of HQET. For lack of space we do not show more examples. We only note that the coefficient of the $1 / z^{n}$ terms in naive fits to the finite mass results are roughly of order unity.

Of course, finite mass lattice QCD results have been compared to the static limit over the years, see for example [27, 3, 4, 28, 29, 30, 31, 32, 33, 34, 35, 36, 37] and references therein. The new quality of the tests just discussed is that the composite fields were renormalized nonperturbatively throughout and that, by considering a small volume, the continuum limit could be taken at large quark masses.

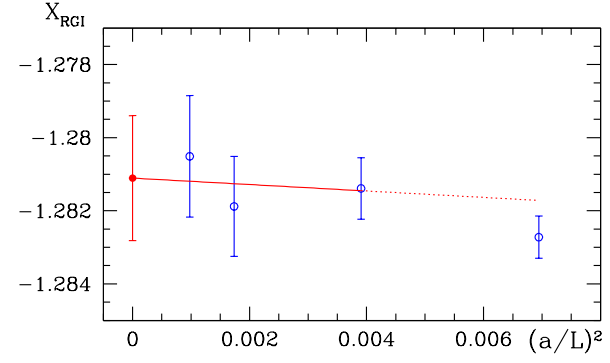

4: Continuum extrapolation of $X_{\mathrm{RGI}}[19]$.

\subsection{Beyond the leading order: the need for non-perturbative conversion functions}

Both from looking at Fig. 3 and from just a naive estimate of $\Lambda / M_{\mathrm{b}}$, one expects that the effective theory has to be implemented beyond the leading order in $1 / M$ to reach an acceptable precision in this expansion. However, if one wants to do this consistently, i.e. one wants to obtain the true coefficients in the expansion, the leading order conversion functions such as $C_{\mathrm{PS}}$ have to be known non-perturbatively. This general problem in the determination of power corrections in QCD is seen by considering the error made in eq. (13) (or eq. (11)) when the anomalous dimension has been computed at $l$ loops and $C_{\text {match }}$ at $l-1$ loop order. The conversion function $C_{\mathrm{PS}}$ is then known up to an error

$$
\Delta\left(C_{\mathrm{PS}}\right) \propto\left[\bar{g}^{2}\left(m_{\mathrm{b}}\right)\right]^{l} \sim\left\{\frac{1}{2 b_{0} \ln \left(M_{\mathrm{b}} / \Lambda\right)}\right\}^{l} \stackrel{M_{\mathrm{b}} \rightarrow \infty}{\gg} \frac{\Lambda}{M_{\mathrm{b}}} .
$$


As $m_{\mathrm{b}}$ is made large, this error becomes dominant. Taking a perturbative conversion function and adding power corrections to the leading order effective theory is thus to be regarded as a phenomenological approach, where one assumes that the coefficient of the $\left[\bar{g}^{2}\left(m_{\mathrm{b}}\right)\right]^{l}$ term is small, such that the $\left(\Lambda / M_{\mathrm{b}}\right)^{n}$ corrections dominate over a certain mass interval. Indeed, returning to our example, Fig. 3 indicates that the power corrections are larger than the perturbative ones at $1 / z=0.1 \ldots 0.2$. Nevertheless, it remains a fact that a theoretically consistent evaluation of power corrections requires a fully non-perturbative formulation of the theory including a non-perturbative matching to QCD.

\section{NON-PERTURBATIVE FORMULATION OF HQET}

The discussion in this section summarizes the main points of [8]. We regularize the theory on a space-time lattice. In the $1 / m_{\mathrm{b}}$ part of the Lagrangian,

$$
\begin{aligned}
\mathscr{L}^{(1)}(x)= & \sum_{i} \omega_{i}^{(1)} \mathscr{L}_{i}^{(1)}(x), \\
& \mathscr{L}_{1}^{(1)}=\bar{\psi}_{\mathrm{h}}(-\sigma \cdot \mathbf{B}) \psi_{\mathrm{h}}, \quad \mathscr{L}_{2}^{(1)}=\bar{\psi}_{\mathrm{h}}\left(-\frac{1}{2} \mathbf{D}^{2}\right) \psi_{\mathrm{h}}
\end{aligned}
$$

the chromo-magnetic field $\mathbf{B}$ and the 3-d Laplacian $\mathbf{D}^{2}$ are then discretized in the standard way. Details will be irrelevant for our discussion. The coefficients $\omega_{i}^{(1)}$ are functions of the bare coupling $g_{0}$ as well as the mass of the heavy quark. They have to be determined such as to match the effective theory to QCD. Matching at the classical level fixes

$$
\omega_{1}^{(1)}=1 / m_{\mathrm{b}}+\mathrm{O}\left(g_{0}^{2}\right)=\omega_{2}^{(1)} .
$$

Furthermore, we note that also in eq. (4) a dimension 4 composite field with coefficient $\propto 1 / m_{\mathrm{b}}+\mathrm{O}\left(g_{0}^{2}\right)$ has to be added on the r.h.s. when $1 / m_{\mathrm{b}}$ corrections are considered. As an additional essential ingredient in the formulation of the effective theory we always expand the formal weight in the path integral, $\exp \left(\sum_{x}-\left(\mathscr{L}_{\text {stat }}(x)+\mathscr{L}^{(1)}(x)+\ldots\right)\right)$, in a power series in $1 / m_{\mathrm{b}}$. The correlation functions are then defined by

$$
\langle\mathscr{O}\rangle=\frac{1}{Z} \int D[\varphi] \mathscr{O}[\varphi] \exp \left(-a^{4} \sum_{\mathbf{x}} \mathscr{L}_{\text {stat }}(\mathbf{x})\right) \times\left\{1-a^{4} \sum_{x} \mathscr{L}^{(1)}(x)+\ldots\right\}
$$

where $\varphi$ denotes collectively the fields of the theory and the denominator $Z$ insures $\langle 1\rangle=1$. The higher order terms in the Lagrangian then appear only as insertions into the correlation functions of the static effective theory. The latter is renormalizable by power counting and as a result also the effective theory truncated at any finite order in $1 / m_{\mathrm{b}}$ is renormalizable. With higher dimensional operators in the exponential, as in NRQCD, this would not be the case. For the lattice theory renormalizability is important because it means that the continuum limit exists and is independent of the details of the lattice formulation (universality). 


\subsection{Power divergencies}

The coefficients $\delta m, \omega_{i}^{(1)}$ in eq. (2) and eq. (21) have a regular expansion in the bare coupling $g_{0}^{2}$. Still, perturbative precision is in general insufficient, since operators of higher dimensions mix with those of lower dimension, e.g.

$$
\mathscr{O}_{\mathrm{R}}^{\mathrm{d}=5}=\sum_{k} z_{k} \mathscr{O}_{k}^{\mathrm{d}=5}+\sum_{k} c_{k} \mathscr{O}_{k}^{\mathrm{d}=4}, \quad c_{k}=\frac{c_{k}^{(0)}+c_{k}^{(1)} g_{0}^{2}+\ldots}{a} .
$$

Since the lattice spacing decreases as $a \sim \exp \left(-1 /\left(2 b_{0} g_{0}^{2}\right)\right)$ for small bare gauge coupling $g_{0}$, a truncation of the perturbative series leaves terms undetermined which diverge as the lattice spacing goes to zero. The origin of this problem is the same as the need for non-perturbative conversion functions, but the consequence is more drastic due to the presence of the hard cutoff in the lattice theory. Without non-perturbative precision for $\delta m, \omega_{i}^{(1)}$, the continuum limit does not exist.

\subsection{Matching strategy}

The definition of the effective theory is essentially given by eq. (24), supplemented by a definition of the effective composite fields. The only missing piece is a practical strategy for ascertaining how the parameters in the Lagrangian and in the effective fields can be determined beyond perturbation theory. At a given order $n$ in the $1 / m_{\mathrm{b}}$-expansion, we denote the parameters in the effective theory by $c_{k}, k=1, \ldots, N_{n}$. Observables, e.g. renormalized correlation functions or energies are denoted by $\Phi_{k}^{\mathrm{HQET}}(L, M)\left(\Phi_{k}^{\mathrm{QCD}}(L, M)\right)$ in the effective theory (in QCD), with the argument $M$ referring to the mass of the heavy quark and $L$ the linear extent of the finite volume. It is then sufficient to impose

$$
\Phi_{k}^{\mathrm{HQET}}\left(L_{0}, M\right)=\Phi_{k}^{\mathrm{QCD}}\left(L_{0}, M\right), \quad k=1, \ldots, N_{n} .
$$

to determine all parameters $\left\{c_{k}, k=1, \ldots, N_{n}\right\}$ in the effective theory. Observables used originally to fix $\left\{c_{k}, k=1, \ldots, N_{\mathrm{f}}\right\}$, the parameters of $\mathrm{QCD}$, may be amongst these $\Phi_{k}^{\mathrm{QCD}}$. The matching conditions, eq. (26), define the set $\left\{c_{k}\right\}$ for any value of the lattice spacing (or equivalently bare coupling). Here, a typical choice is $L_{0} \approx 0.2 \ldots 0.4 \mathrm{fm}$, since in such a volume the r.h.s. of the equation can be evaluated, see Sect. 2.1. In practice, the parameters of the effective theory are then determined at rather small lattice spacings in a range of $a=0.01 \mathrm{fm}$ to $a=0.04 \mathrm{fm}$. Large volumes as they are needed to compute the physical mass spectrum or matrix elements then require very large lattices $(L / a>50)$. A further step is needed to bridge the gap to practicable lattice spacings. A well-defined procedure is as follows: First we assume that all observables $\Phi_{k}^{\mathrm{HQET}}(L, M)$ have been made dimensionless by multiplication with appropriate powers of $L$. Next, we define step scaling functions [38], $F_{k}$, by

$$
\Phi_{k}^{\mathrm{HQET}}(s L, M)=F_{k}\left(\left\{\Phi_{j}^{\mathrm{HQET}}(L, M), j=1, \ldots, N_{n}\right\}\right), \quad k=1, \ldots, N_{n},
$$


where typically one uses scale changes of $s=2$. These dimensionless functions describe the change of the complete set of observables $\left\{\Phi_{k}^{\mathrm{HQET}}\right\}$ under a scaling of $L \rightarrow s L$. In order to compute them one selects a lattice with a certain resolution $a / L$. The specification of $\Phi_{j}^{\mathrm{HQET}}(L, M), j=1, \ldots, N_{n}$, then fixes all (bare) parameters of the theory. The 1.h.s. of eq. (27) is now computed, keeping the bare parameters fixed while changing $L / a \rightarrow L^{\prime} / a=s L / a$. The values for the continuum $F_{k}$ can then be beached by extrapolating the resulting lattice numbers to $a / L \rightarrow 0$.

After repeating this step two or three times with $s=2$, lattice spacings appropriate for infinite volume computations will be reached.

\subsection{Example: the mass of the b-quark at lowest order}

For illustration purposes we consider a simple example here, the computation of the b-quark mass, starting from the observed B-meson mass. Already at the lowest order in $1 / m_{\mathrm{b}}$ the mixing of operators of different dimensions is relevant in this case: $\bar{\psi}_{\mathrm{h}} D_{0} \psi_{\mathrm{h}}$ mixes with $\bar{\psi}_{\mathrm{h}} \psi_{\mathrm{h}}$. Hence $\delta m=\left(c_{1} g_{0}^{2}+c_{2} g_{0}^{4}+\ldots\right) / a$, the coefficient of $\bar{\psi}_{\mathrm{h}} \psi_{\mathrm{h}}$ in the Lagrangian eq. (2), has to be determined (or eliminated) non-perturbatively. In eq. (26) we have $n=0, N_{0}=N_{\mathrm{f}}+1$ and we omit the discussion of the choices for $\Phi_{k}$, $k=1, \ldots, N_{\mathrm{f}}$ which fix the bare light quark masses and coupling (both in QCD and HQET). Obviously any energy in the b-sector will do to fix $\delta m$. We choose [8, 39] ${ }^{4}$

$$
\Gamma(L, M)=\frac{1}{2 a} \ln \left[f_{\mathrm{A}}\left(x_{0}-a\right) / f_{\mathrm{A}}\left(x_{0}+a\right)\right] \quad\left(x_{0} / L \text { fixed }\right)
$$

and we require eq. (26) where for $k=N_{\mathrm{f}}+1$ we identify

$$
\Phi_{N_{\mathrm{f}}+1}^{\mathrm{QCD}}(L, M) \equiv L \Gamma(L, M), \quad \Phi_{N_{\mathrm{f}}+1}^{\mathrm{HQET}}(L, M) \equiv L\left(\Gamma_{\text {stat }}(L)+m\right),
$$

Here $m$ represents the quark mass that was removed from all energies in defining the effective theory such that the $m \rightarrow \infty$ limit exists and $\Gamma_{\text {stat }}$ refers to eq. (28) at the lowest order in $1 / M$. The relevant part of eq. (27) can then be written in the simple form,

$$
\begin{aligned}
\Phi_{N_{\mathrm{f}}+1}^{\mathrm{HQET}}(2 L, M) & =2 \Phi_{N_{\mathrm{f}}+1}^{\mathrm{HQET}}(L, M)+\sigma_{\mathrm{m}}\left(\bar{g}^{2}(L)\right), \\
\sigma_{\mathrm{m}}\left(\bar{g}^{2}(L)\right) & \equiv 2 L\left[\Gamma_{\text {stat }}(2 L)-\Gamma_{\text {stat }}(L)\right] .
\end{aligned}
$$

In $\sigma_{\mathrm{m}}$ the divergent $\delta m$ (as well as the mass shift $m$ ) cancel. It is independent of the mass. We now see immediately that

$$
m_{\mathrm{B}}=\underbrace{E_{\text {stat }}-\Gamma_{\text {stat }}\left(L_{2}\right)}_{a \rightarrow 0 \text { in HQET }}+\underbrace{\Gamma_{\text {stat }}\left(L_{2}\right)-\Gamma_{\text {stat }}\left(L_{0}\right)}_{a \rightarrow 0 \text { in HQET }}+\underbrace{\Gamma\left(L_{0}, M_{\mathrm{b}}\right)}_{a \rightarrow 0 \text { in QCD }}+\mathrm{O}\left(\Lambda^{2} / M_{\mathrm{b}}\right) .
$$

Here, $E_{\text {stat }}=\lim _{L \rightarrow \infty} \Gamma_{\text {stat }}(L)$ is the infinite volume energy of a B-meson in static approximation. It is often called the static binding energy. The whole strategy is illustrated

${ }^{4}$ In practice $\Gamma$ is replaced by the spin averaged energy [8, 39]. 
in Fig. 5. As indicated in eq. (32), the continuum limit can be taken in each individual step; a numerical example is shown in Fig. 6

\begin{tabular}{|c|c|c|c|c|}
\hline experiment & & & & Lattice with $a m_{\mathrm{b}} \ll 1$ \\
\hline$m_{\mathrm{B}}=5.4 \mathrm{GeV}$ & & & & $\Gamma\left(L_{0}, M\right)$ \\
\hline & & $L_{i}=2^{i} L_{0}$ & & \\
\hline$\Gamma_{\text {stat }}\left(L_{2}\right)$ & $\overline{\sigma_{\mathrm{m}}\left(u_{1}\right)}$ & $\Gamma_{\text {stat }}\left(L_{1}\right)$ & $\overline{\sigma_{\mathrm{m}}\left(u_{0}\right)}$ & $\Gamma_{\text {stat }}\left(L_{0}\right)$ \\
\hline
\end{tabular}

FIGURE 5. Connecting experimental observables to renormalized HQET. $\Gamma_{\text {stat }}$ is a renormalized quantity in HQET and $\sigma_{\mathrm{m}}\left(\bar{g}^{2}(L)\right)$ connects $\Gamma_{\text {stat }}(L)$ and $\Gamma_{\text {stat }}(2 L)$.

After obtaining all pieces in eq. (32), the equation is numerically solved for $z_{\mathrm{b}}=M_{\mathrm{b}} L_{0}$. Since also the size of $L_{0}$ in units of $r_{0} \approx 0.5 \mathrm{fm}$ [40] is known, one can quote (remember $m_{\mathrm{b}}$ is in the $\overline{\mathrm{MS}}$ scheme at scale $m_{\mathrm{b}}$ )

$$
r_{0} M_{\mathrm{b}}=16.12(24)(15) \rightarrow M_{\mathrm{b}}=6.36(10)(6) \mathrm{GeV}, \quad m_{\mathrm{b}}=4.12(7)(4) \mathrm{GeV} .
$$

We emphasize that this result is in the quenched approximation but includes the lowest non-trivial order in $1 / m_{\mathrm{b}}$. An estimate of the associated $\mathrm{O}\left(\Lambda^{2} / M_{\mathrm{b}}\right)$ uncertainty is not included in the errors shown. Our discussion mainly serves to illustrate the potential of the approach in an example where the power divergent mixing needed to be solved non-perturbatively.

\section{PERSPECTIVES}

Non-perturbative HQET at the leading order in $1 / m$ has reached a satisfactory status, with the b-quark mass [8] and the $\mathrm{B}_{\mathrm{s}}$-meson decay constant $[6,41]$ known in the continuum limit of the quenched approximation. Their precision can and will still be improved. Applying these methods to the theory with dynamical fermions is straight forward; "only" the usual problems with the light quarks have to be solved. By themselves such lowest order results are not expected to have an interesting precision for phenomenological applications, but

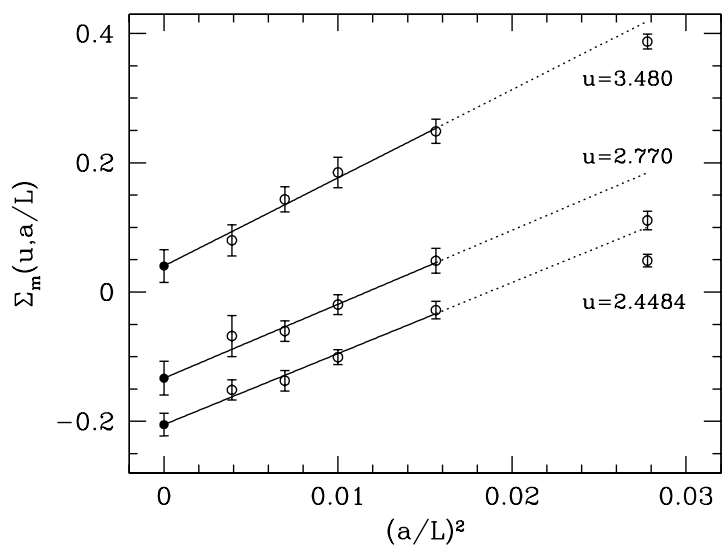

6: Extrapolation $\Sigma_{\mathrm{m}}(u, a / L)=\sigma_{\mathrm{m}}(u)+c a^{2} / L^{2}$ certainly they can constrain the large mass behavior computed with other methods $[27,3,4,28,29,30,31,32,33,34,35,36,37]$.

More interestingly, also $1 / m_{\mathrm{b}}$ corrections can, in principle, be computed in the effective theory. Here, details of the necessary numerical steps have not yet been implemented but the very first tests have been encouraging [42]. Another relevant technical advance has been the realization that a change of the regularization details allows to achieve much 
better statistical errors in HQET, while keeping the discretization errors small [41]. In summary, we believe that all ingredients exist which are needed to apply HQET beyond the leading order in $1 / m_{\mathrm{b}}$.

\section{ACKNOWLEDGMENTS}

I would like to thank my colleagues S. Dürr, M. Della Morte, A. Jüttner, J. Rolf, and in particular J. Heitger for a pleasant collaboration in developing non-perturbative HQET. I am grateful to $\mathrm{H}$. Simma for useful comments on this manuscript.

\section{REFERENCES}

1. E. Eichten, Nucl. Phys. Proc. Suppl., 4, 170 (1988).

2. E. Eichten, Nucl. Phys. Proc. Suppl., 20, 475-487 (1991).

3. R. Sommer, Phys. Rept., 275, 1-47 (1996), hep-lat/9401037

4. H. Wittig, Int. J. Mod. Phys., A12, 4477-4538 (1997), hep-lat/9705034

5. L. Maiani, G. Martinelli, and C. T. Sachrajda, Nucl. Phys., B368, 281-292 (1992).

6. J. Heitger, M. Kurth, and R. Sommer, Nucl. Phys., B669, 173-206 (2003), hep-lat / 0302019

7. J. Heitger, and R. Sommer, Nucl. Phys. Proc. Suppl., 106, 358-360 (2002), hep-lat/ 0110016

8. J. Heitger, and R. Sommer, JHEP, 02, 022 (2004), hep-lat/0310035

9. M. Neubert, Phys. Rept., 245, 259-396 (1994), hep-ph/9306320

10. T. Mannel (1996), hep-ph/9606299

11. B. A. Thacker, and G. P. Lepage, Phys. Rev., D43, 196-208 (1991).

12. D. J. Broadhurst, and A. G. Grozin, Phys. Lett., B267, 105-110 (1991), hep-ph/9908362

13. M. A. Shifman, and M. B. Voloshin, Sov. J. Nucl. Phys., 45, 292 (1987).

14. H. D. Politzer, and M. B. Wise, Phys. Lett., B206, 681 (1988).

15. K. G. Chetyrkin, and A. G. Grozin (2003), hep-ph/0303113

16. X. Ji, and M. J. Musolf, Phys. Lett., B257, 409 (1991).

17. D. J. Broadhurst, and A. G. Grozin, Phys. Rev., D52, 4082-4098 (1995), hep-ph/9410240

18. V. Gimenez, Nucl. Phys., B375, 582-624 (1992).

19. J. Heitger, A. Jüttner, R. Sommer, and J. Wennekers, JHEP, 11, 048 (2004), hep-ph/ 0407227

20. M. Lüscher, R. Narayanan, P. Weisz, and U. Wolff, Nucl. Phys., B384, 168-228 (1992), hep-lat/9207009

21. S. Sint, Nucl. Phys., B421, 135-158 (1994), hep-lat/9312079

22. M. Kurth, and R. Sommer, Nucl. Phys., B597, 488-518 (2001), hep-lat/0007002

23. S. Capitani, M. Lüscher, R. Sommer, and H. Wittig, Nucl. Phys., B544, 669 (1999), hep-lat/9810063

24. M. E. Luke, and A. V. Manohar, Phys. Lett., B286, 348-354 (1992), hep-ph/9205228

25. W. Kilian, and T. Ohl, Phys. Rev., D50, 4649-4656 (1994), hep-ph/9404305

26. R. Sundrum, Phys. Rev., D57, 331-336 (1998), hep-ph/9704256

27. C. Alexandrou, et al., Z. Phys., C62, 659-668 (1994), hep-lat/9312051

28. A. X. El-Khadra, A. S. Kronfeld, P. B. Mackenzie, S. M. Ryan, and J. N. Simone, Phys. Rev., D58, 014506 (1998), hep-ph/9711426

29. S. Aoki, et al., Phys. Rev. Lett., 80, 5711-5715 (1998).

30. C. W. Bernard, et al., Phys. Rev. Lett., 81, 4812-4815 (1998), hep-ph/9806412

31. D. Becirevic, et al., Phys. Rev., D60, 074501 (1999), hep-lat/9811003

32. A. Ali Khan, et al., Phys. Rev., D64, 034505 (2001), hep-lat/0010009

33. K. C. Bowler, et al., Nucl. Phys., B619, 507-537 (2001), hep-lat/ 0007020

34. L. Lellouch, and C. J. D. Lin, Phys. Rev., D64, 094501 (2001), hep-ph/0 011086

35. S. M. Ryan, Nucl. Phys. Proc. Suppl., 106, 86-97 (2002), hep-lat/0111010

36. G. M. de Divitiis, M. Guagnelli, R. Petronzio, N. Tantalo, and F. Palombi, Nucl. Phys., B675, 309332 (2003), hep-lat/0305018 
37. G. M. de Divitiis, M. Guagnelli, F. Palombi, R. Petronzio, and N. Tantalo, Nucl. Phys., B672, 372386 (2003), hep-lat/0307005

38. M. Lüscher, P. Weisz, and U. Wolff, Nucl. Phys., B359, 221-243 (1991).

39. J. Heitger, and J. Wennekers, JHEP, 02, 064 (2004), hep-lat/0312016

40. R. Sommer, Nucl. Phys., B411, 839 (1994), hep-lat/9310022

41. M. Della Morte, et al., Phys. Lett., B581, 93-98 (2004), hep-lat/ 0307021

42. S. Dürr, A. Jüttner, J. Rolf, and R. Sommer (2004), hep-lat/ 0409058 